\title{
Unfolding, Fibre Bundles, Color Breaking and Gauge Invariance
}

\author{
E.C.G. SUDARSHAN
}

Center for Particle Theory and Department of Physics, The University of Texas at Austin, Austin, Texas 78712, U.S.A.

\section{Introduction}

Newton's Laws of Motion and their natural generalizations are most succinctly embodied in the action principle in the Lagrangian form:

$$
\delta \int L(q, \dot{q}) \mathrm{d} t=0
$$

In the generic case the variational Euler-Lagrange equations are second order nonlinear equations which would express the accelerations $\ddot{q}$ in terms of the generalized velocities $\dot{q}$ and positions $q$. It is more convenient to pass from these Lagrangian equations to the Hamiltonian equations with

$$
\begin{aligned}
& p=\partial L / \partial \dot{q} ; \quad H(p, q)=p \dot{q}-L(q, \dot{q}) \\
& \dot{p}=-\partial H / \partial q ; \quad \dot{q}=\partial H / \partial p
\end{aligned}
$$

making use of a Legendre transformation. These are now first order equations with the phase space variables $\xi=\{q, p\}$ being treated as the configuration of the system. For a dynamic picture of the evolution it is most natural to deal with the Hamiltonian form but the action principle is expressed in the Lagrangian form.

It is a natural question to ask: Given the Hamiltonian equations, which can be written uniformly, in the form:

$$
\dot{f}(\xi)=[f(\xi), H(\xi)]_{\text {P.b. }}
$$

in terms of the Poisson bracket, could we formulate an action principle also in phase space? In other words could we get an action principle without ever going to the Lagrangian form? In this context we must allow for the possibility that the coordinatization of phase space that is employed cannot be done globally but must be done by the use of overlapping patches.

To get the phase space form of the action principle we define the action along a phase trajectory by the integral

$$
\psi[\Gamma]=\int_{t_{1}}^{t_{2}}(p \dot{q}-H(q, p)) \mathrm{d} t=\int_{\Gamma}(p \mathrm{~d} q-H \mathrm{~d} t) .
$$


The Hamiltonian principle in Hamiltonian form is then the statement that

$$
\delta \psi=0
$$

for infinitesimal variations of $\Gamma$. The equations of motion are the Hamiltonian equations with the Poisson brackets being defined by

$$
[F(q, p), G(q, p)]=\frac{\partial F}{\partial q} \frac{\partial G}{\partial p}-\frac{\partial F}{\partial p} \frac{\partial G}{\partial q}
$$

The symplectic structure is given by the 2-form on the cotangent bundle $T^{*} \mathrm{Q}$ ( $\mathrm{Q}$ being the configuration space):

$$
\omega=\mathrm{d} A ; \quad A=p \mathrm{~d} q .
$$

This 2 -form is closed and exact.

A more general symplectic structure [1] corresponds to the generalized Poisson bracket (g.P.b.)

$$
[F(\xi), G(\xi)]=\omega^{\mu \nu}(\xi) \frac{\partial F}{\partial \xi^{\mu}} \frac{\partial G}{\partial \xi^{\nu}}
$$

the associated 2-form is:

$$
\begin{aligned}
& \omega=\mathrm{d} A=-\frac{1}{2} \omega_{\mu \nu}(\xi) \mathrm{d} \xi^{\mu} \wedge \mathrm{d} \xi^{\nu} ; \\
& \omega_{\mu \nu}(\xi)=\partial A_{\nu}(\xi) / \partial \xi^{\mu}-\partial A_{\mu}(\xi) / \partial \xi^{\nu} ; \quad \omega^{\mu \nu} \omega_{\nu \lambda}=\delta_{\lambda}^{\mu} .
\end{aligned}
$$

For this g.P.b. the Jacobi identity holds since the 2-form, being exact, is closed:

$$
\partial_{\lambda} \omega_{\mu \nu}+\partial_{\nu} \omega_{\lambda \mu}+\partial_{\mu} \omega_{\nu \lambda}=0
$$

\section{Nonexact 2-forms and path spaces [2]}

However if we started out with a generalized Poisson bracket with a closed 2-form and the Hamiltonian equations, we see that since this involves only the 2-form, its closure ensures their consistency. A genuinely new situation arises if the 2 -form $\omega$ is closed but not exact: this would mean that there is no global 1-form $A$ such that $\omega=\mathrm{d} A$. In such a case we can construct an action functional and a variational principle from which these equations follow:

Let us make the requirement that the phase space is path-connected and let $\xi_{0}{ }^{\mu}$ be a fixed point in phase space. For a trajectory $\xi^{\mu}(t), t_{1} \leq t \leq t_{2}$ define

$$
\gamma^{\mu}(0, t)=\xi_{0}{ }^{\mu} ; \quad \gamma^{\mu}(1, t)=\xi^{\mu}(t)
$$

and let $\gamma^{\mu}(\sigma, t), 0 \leq \sigma \leq 1$ be a smooth curve for each $\sigma$. Then $\gamma^{\mu}(\sigma, t)$ sweeps out a smooth surface $\mathrm{S}$ which could be thought of as being swept by a curve starting at $\xi_{0}{ }^{\mu}$ and ending at $\xi^{\mu}(t)$ as $t$ varies. We 
can now define an action functional

$$
\begin{aligned}
\Psi[\mathrm{S}] & =\int_{t_{1}}^{t_{1}} \mathrm{~d} t \int_{0}^{1} \mathrm{~d} \sigma \omega_{\mu \nu}(\gamma) \frac{\partial \gamma^{\mu}}{\partial \sigma} \frac{\partial \gamma^{\nu}}{\partial t}-\int_{t_{1}}^{t_{2}} H(\xi(t)) \mathrm{d} t \\
& =\int_{\mathrm{S}} \omega-\int_{\Gamma} H \mathrm{~d} t .
\end{aligned}
$$

This functional depends only on the boundary $\partial S$ of $S$ and not on $S$ itself as long as $S$ undergoes continuous change since $\omega$ is closed. However there is no global way of expressing $\Psi[S]$ explicitly in terms of $\partial \mathrm{S}$ alone, much less on the part $\Gamma$ of $\partial \mathrm{S}$.

Usually we think of the Poisson brackets as an expression of the kinematics; and the Hamiltonian as embodying the dynamics. Here there is a reversal of roles in that $H$ is not changed while the symplectic 2-form $\omega^{\mu \nu}$ and its Lagrange bracket inverse $\omega_{\mu \nu}$ are changed, thus geometrizing dynamics.

\section{Unfolding [2]}

If we further assume that even in this generalized case there is a well-defined configuration space $\mathrm{Q}$ with local coordinate $q^{j}$ and other variables $p_{j}$, where the index $j$ runs from 1 to $n$. The generalized bracket should then split in the form

$$
\begin{aligned}
& \omega^{j k}=0, \quad \omega^{j, k+n}=-\omega^{j+n, k}=\delta_{j k} \\
& \omega^{j+n, k+n}=B_{j k}(q) .
\end{aligned}
$$

Then the inverse $\omega_{\mu \nu}$ is the 2 -form with components

$$
\begin{aligned}
& \omega_{j k}=B_{j k}(q) ; \quad \omega_{j+n, k+n}=0 ; \\
& \omega_{j, k+n}=-\omega_{j+n, k}=-\delta_{j k} .
\end{aligned}
$$

The closure property of the 2-form, which guarantees Jacobi identity requires

$$
\frac{\partial}{\partial q^{j}} B_{k l}+\frac{\partial}{\partial q_{k}} B_{l j}+\frac{\partial}{\partial q^{l}} B_{j k}=0
$$

If the 2 -form is exact there is a 1 -form $A_{j}(k)$ such that

$$
B_{j k}(q)=\frac{\partial}{\partial q_{j}} A_{k}(q)-\frac{\partial}{\partial q_{k}} A_{j}(q)
$$

but for a nonexact 2 -form no such global definition exists. 
For this special class of systems one can go back to a Lagrangian description by the standard recipe

$$
L(q, \dot{q})=\dot{q} p-H(q, p)
$$

with the Lagrangian equations

$$
\frac{\mathrm{d}}{\mathrm{d} t} \frac{\partial L}{\partial \dot{q}^{j}}-\frac{\partial L}{\partial q^{j}}=B_{j k}(q) \dot{q}^{k} .
$$

Assuming $\mathrm{Q}$ is path-connected, introduce the family of curves

$$
\begin{aligned}
& \beta^{j}(\sigma, t): 0 \leq \sigma \leq 1 ; \quad t_{1}<t<t_{2} \\
& \beta^{j}(0, t)=q_{0}^{j} ; \quad \beta^{j}(1, t)=q^{j}(t) .
\end{aligned}
$$

Then we can have the action functional

$$
\Phi(\mathrm{S})=\int_{t_{1}}^{t_{2}} \mathrm{~d} t \int_{0}^{1} \mathrm{~d} \sigma B_{j k}(\beta) \frac{\partial \beta^{j}}{\partial \sigma} \frac{\partial \beta^{k}}{\partial t}+\int_{t_{1}}^{t_{2}} \mathrm{~d} t L(q, \dot{q})
$$

So in this case we may view this to be the action function for a generalized Lagrangian system whose configuration space is not $Q$ but the path space of $Q$ consisting of continuous paths in $Q$ form a fixed point $q_{0}^{j}$ to the points of $\mathrm{Q}$. Such a description contains a great number of redundant variables which do not appear in the final equations of motion for the accelerations $\ddot{q}$ but which are essential to the formulation of the variational principle.

\section{Monopole-charge system}

The essential point to be recognized is that it is not the path itself that is relevant but the equivalence class of paths, any two paths which can be continuously deformed into each other being considered equivalent. If there are several equivalence classes, the label of the equivalence class is a new configuration label required for complete specification. To overcome the topological obstruction that leads to the nonequivalence, paths can be "unfolded" only in a larger manifold which will contain local ignorable coordinates.

The electric charge-Dirac monopole [3] system provides the simplest nontrivial example. The equations of motion are

$$
m \ddot{x}^{j}=\frac{e g}{r^{3}} \varepsilon^{j k l} \dot{x}^{k} x^{l}
$$

The corresponding Hamiltonian equations

$$
\dot{x}^{j}=\frac{p^{j}}{m} ; \quad p^{j}=\frac{e g}{m r^{3}} \varepsilon^{j k l} p^{k} x^{l}
$$


would be obtained from the Hamiltonian

$$
H=p^{2} / 2 m
$$

with the generalized Poisson brackets:

$$
\begin{aligned}
& \left\{x^{j}, x^{k}\right\}=0 ; \quad\left\{x^{j}, p^{k}\right\}=\delta^{j k} \\
& \left\{p^{j}, p^{k}\right\}=-\frac{e g}{r^{3}} \varepsilon^{l j k} x^{l} .
\end{aligned}
$$

The configuration space $Q$ is $\mathbb{R}^{3}-\{0\}$. The associated 2-form

$$
B=\frac{1}{2} B_{j k}(x) \mathrm{d} x^{j} \wedge \mathrm{d} x^{k}=-e g \sin \theta \mathrm{d} \theta \wedge \mathrm{d} \phi
$$

is closed but not exact. This is easily recognized as a multiple of the volume on the 2 -sphere $S^{2}$,

$$
\int_{\mathrm{s}^{2}} B=-4 \pi e g
$$

\section{Dynamics on sections [4]}

The equations of motion of the charge-monopole system considered in the last section was first considered in the context of quantum theory by Dirac [3] who showed that it entails the concept of nonintegrable phases which in turn leads to the quantization of the product e.g. of charge and monopole strength. Saha [3] pointed out that the conserved angular momentum of the charge-monopole system has an intrinsic angular momentum of $\frac{1}{2} \hbar$ which must be added to orbital angular momentum.

In the context of non-Abelian gauge theories accompanied by spontaneous symmetry breaking new kinds of monopoles arise. In such theories, once the symmetry has broken down there arise distinct sectors in which the asymptotic nonvanishing field configurations are topologically nonequivalent; and in some of these there are long-range generalized magnetic fields reminiscent of the Dirac monopole. But unlike the Dirac monopole which has a singularity at the origin, in these theories there is only a soft field configuration which is persistent but nonsingular at the origin. It is this twin generalization of non-Abelian symmetry groups and topologically inequivalent configurations that generate the richness of the non-Abelian monopoles. Let us briefly survey the Dirac monopole both for its own sake as illustrating a theory requiring nontrivial manifolds globally but also to set the scene for the non-Abelian monopoles.

Given the equations of motion we mentioned above:

$$
m \ddot{x}_{j}=\frac{e g}{r^{3}} \varepsilon_{j k l} \dot{x}^{k} x^{l}=e B_{j k}(x) \dot{x}^{k}
$$

with 


$$
B_{j k}(x)=\frac{g}{r^{3}} \varepsilon_{j k l} x^{l}
$$

if we attempt to construct a vector potential $W^{j}(x)$ we find that no global solution exists for a $W^{j}$ which satisfies

$$
B_{j h}(x)=\partial_{j} W_{k}(x)-\partial_{k} W_{j}(x)
$$

If we denote by $\Sigma$ the sphere $S^{2}$ we can define $W^{N}{ }_{j}$ on a contractible open set $\Sigma_{\mathrm{N}}$ which may be thought of as the sphere $S^{2}$ with a neighborhood of the South pole removed [4]. Equally well we can make a different choice $W^{\mathrm{s}}$, defined on a contractible open set $\Sigma_{\mathrm{s}}$ with a neighborhood of the North pole being removed for $S^{2}$. In the region of overlap $\Sigma_{N} \cap \Sigma_{S}$ the two vector potentials are connected by

$$
\begin{aligned}
& W_{j}^{\mathrm{N}}(x)=h_{T}^{-1}(x)\left(W_{j}^{\mathrm{s}}(x)-\frac{\mathrm{i}}{e} \partial_{j}\right) h_{T}(x) ; \\
& B^{\mathrm{N}}{ }_{j k}(x)=B_{j k}^{\mathrm{S}}(x),
\end{aligned}
$$

where $h_{T}(x)$ is a phase factor chosen to be

$$
h_{T}(x)=\mathrm{e}^{\mathrm{i} \phi},
$$

where $\phi$ is the azimuthal angle. There is no global definition possible for $W_{j}(x)$ as long as we stay with the configuration space which is the direct product of the open set $0<r<\infty$ with $S^{2}$. We must construct a fibre bundle on which the dynamics can be globally defined.

Now let us proceed to consider a test particle carrying internal symmetry ("isospin") charge $I_{a}$ which is a vector in the space of the adjoint representation of the symmetry group $\mathrm{H}$ with generators $T_{a}$. Then the vector potential $W_{j}$, field strength $G_{j k}$ and the isospin $I$ may be written in the form:

$$
\begin{aligned}
& W_{j}(x)=W_{a j}(x) T_{a} ; \quad G_{j k}(x)=G_{a j k}(x) T_{a} \\
& I=I_{a} T_{a} \\
& G_{j k}(x)=\partial_{j} W_{k}(x)-\partial_{k} W_{j}(x)+\mathrm{i} e\left[W_{j}(x), W_{k}(x)\right] .
\end{aligned}
$$

The Wong equations of motion [6] are:

$$
\begin{aligned}
& m \ddot{x}_{j}=-e \operatorname{Tr}\left(I G_{j k}(x)\right) \dot{x}_{k} \\
& \dot{I}=-i e \dot{x}_{j}\left[W_{j}(x), I\right] .
\end{aligned}
$$

We may alter the description by a gauge transformation:

$$
\begin{aligned}
& W_{j}^{\prime}=g^{-1}(x)\left(W_{j}(x)-\frac{\mathrm{i}}{e} \partial_{j}\right) g(x) ; \\
& G_{j k}^{\prime}=g^{-1}(x) G_{j k}(x) g(x) ; \\
& I^{\prime}=g^{-1}(x) \operatorname{Ig}(x) .
\end{aligned}
$$


Of course, given an external field $G_{j k}$ the isospin $I$ is not a constant but precesses according to the Wong equations. But the isospin can be defined globally as long as the external gauge field configuration is topologically trivial. But as soon as the fields have nontrivial topological properties (like a monopole) the isospin cannot be globally defined. This is in contrast to the Dirac monopole for which the equations of motion were global. The action of the "isospin" group $\mathrm{H}$ is then not something globally defined. We shall discuss this below in connection with color breaking by monopoles; but we see that as far as classical theories are concerned we may work with the equations defined on the mutually overlapping contractible sections $\Sigma_{\mathrm{N}}$ and $\Sigma_{\mathrm{s}}$. It could nevertheless be desirable to construct a formalism where all quantities are defined globally. This is provided by the introduction of fibre bundles. Since the field strength $G$ is totally radial the vector potentials $W$ have no radial components, though the angular components have both radial and angular dependences. The well-defined but distinct $W_{\mathrm{N}}$ and $W_{\mathrm{S}}$ over $\Sigma_{\mathrm{N}}$ and $\Sigma_{\mathrm{S}}$ related through $h_{T}(x)$ can be obtained by the specialization of a connection $\Omega$ on a principal H-bundle over the base $S^{2}=\Sigma=\Sigma_{N} \cup \Sigma_{S}$. The locally defined potentials $W_{N}, W_{S}$ arise by restricting the global connection $\Omega$ to the sections $\Sigma_{\mathrm{N}}$ and $\Sigma_{\mathrm{s}}$. The precise form of $\Omega$ depends on the group $\mathrm{H}$.

\section{Fibre bundles and connections $[7,8]$}

As a specific example of such a connection we choose the transition function

$$
h_{T}(x)=\mathrm{e}^{\mathrm{i} \phi T}
$$

where $T$ is a generator of the Lie algebra $\mathbf{H}$. Then the fibre bundle $\mathscr{B}$ over the sphere $S^{2}$ is obtained from the Cartesian product

$$
\overline{\mathscr{B}}=\mathrm{H} \times \mathrm{SU}(2)=\{(B, u) \mid B \in \mathrm{H}, u \in \mathrm{SU}(2)\}
$$

by introducing the equivalence relation

$$
(B, u) \sim\left(\mathrm{e}^{-\mathrm{i} \alpha T} B, u \mathrm{e}^{\mathrm{i} \alpha \sigma_{3}}\right), \quad 0 \leq \alpha \leq 2 \pi .
$$

The product $\overline{\mathscr{B}}$ equipped with this equivalence relation is the fibre bundle $\mathscr{B}=\langle B, u\rangle$. The projection

$$
\pi=\mathscr{B} \rightarrow \mathrm{S}^{2}
$$

is defined by

$$
\pi(\mathscr{B})=\frac{1}{2} \operatorname{Tr}\left(\sigma u \sigma_{3} u^{-1}\right)=\hat{x} .
$$

For $\Sigma_{\mathrm{N}}$ define:

$$
u_{\mathrm{N}}(x)=\exp \left(-\frac{1}{2} \mathrm{i} \phi \sigma_{3}\right) \cdot \exp \left(-\frac{1}{2} \mathrm{i} \theta \sigma_{2}\right) \cdot \exp \left(\frac{1}{2} \mathrm{i} \phi \sigma_{3}\right)
$$

and for $\Sigma_{\mathbf{s}}$ :

$$
u_{\mathrm{S}}(x)=\exp \left(-\frac{1}{2} \mathrm{i} \phi \sigma_{3}\right) \cdot \exp \left(-\frac{1}{2} \mathrm{i} \theta \sigma_{2}\right) \cdot \exp \left(-\frac{1}{2} \mathrm{i} \phi \sigma_{3}\right)
$$


Note that $u_{\mathrm{N}}$ is defined everywhere except at the South pole and $u_{\mathrm{S}}$ everywhere except at the North pole. The global action of $\mathrm{H}$ on $\mathscr{B}$ is:

$$
\langle B, U\rangle \in \mathscr{B} \rightarrow\left\langle B B^{\prime}, u\right\rangle \in \mathscr{B} .
$$

The connection $\Omega$ should be defined over $\mathscr{B}$ rather than $\overline{\mathscr{B}}$ and should transform under the global action of $\mathrm{H}$ according to

$$
\begin{aligned}
& \Omega\left(B B^{\prime}, u\right)=B^{\prime-1} \Omega(B, u) B^{\prime}-\frac{\mathrm{i}}{e} B^{\prime-1} B^{\prime} \\
& \Omega\left(\mathrm{e}^{-\mathrm{i} \alpha T} B, u \mathrm{e}^{\mathrm{i} \alpha \sigma_{3}}\right)=\Omega(B, u) .
\end{aligned}
$$

In addition to these requirements we wish to impose the condition of spherical symmetry appropriate to a monopole:

$$
\Omega(B, u)=\Omega\left(B, u^{\prime} u\right), \quad u^{\prime} \in \mathrm{SU}(2)
$$

Here $u^{\prime}$ is a rigid rotation independent of $x$. The solution [9] satisfying all these three conditions is

$$
\Omega(B, u)=-\frac{\mathrm{i}}{e} B^{-1} \mathrm{~d} B-\frac{\mathrm{i}}{e} B^{-1} T B \cdot \frac{1}{2} \operatorname{Tr}\left(\sigma_{3} u^{-1} \mathrm{~d} u\right)
$$

A global Lagrangian for the motion of a particle in the monopole field is given in terms of a global connection $\Omega$ by:

$$
\mathscr{L}=\frac{1}{2} m x^{2}+\mathrm{i} \operatorname{Tr}\left(K B^{-1}\left[\dot{B}+\mathrm{i} e \frac{\Omega(1, u)}{\mathrm{d} t} B\right]\right) .
$$

With the above choice for $\Omega$ the global Lagrangian is

$$
\mathscr{L}=\frac{1}{2} m \dot{r}^{2}+\frac{1}{2} m r^{2} \dot{\hat{x}}^{2}+\mathrm{i} \operatorname{Tr}\left(K B^{-1} \dot{B}\right)+\mathrm{i} \operatorname{Tr}\left(K B^{-1} T B\right) \frac{1}{2} \operatorname{Tr}\left(\sigma_{3} u^{-1} \dot{u}\right) .
$$

It can be shown that the correct equations of motion follow from this Lagrangian provided the isospin is identified according to:

$$
I=B K B^{-1}
$$

\section{Color breaking: Classical theory}

In a non-Abelian monopole field there may be topological obstructions to the definition of the global action of the group $\mathrm{H}$ on the non-Abelian charges of a particle moving in an H-monopole field. A global definition of the action associates with each $h \in \mathrm{H}$ and each $\hat{x} \in \mathrm{S}^{2}$ an element $k(\hat{x}, h) \in \mathrm{H}_{\hat{x}}$ such that

$$
k\left(\hat{x}, h^{\prime}\right) k(\hat{x}, h)=k\left(\hat{x}, h^{\prime} h\right) .
$$


Thus an isomorphism is established between $\mathrm{H}$ and a group $\mathrm{H}_{\hat{x}}$ at $\hat{x}$. These various groups $\mathrm{H}_{\hat{x}}$ are all to be subgroups of a grand group $\mathrm{G}$. For convenience we shall assume that

$$
k(\hat{x}, h)=h, \quad \hat{x}=\text { North pole }
$$

In the section $\Sigma_{\mathrm{N}}$ we may consider a transformation in $\mathrm{G}$ dependent on $\hat{x}$ such that

$$
h \rightarrow k_{\mathrm{N}}(\hat{x}, h)=g_{\mathrm{N}}(\hat{x})^{-1} k(\hat{x}, h) g_{\mathrm{N}}(\hat{x})
$$

is an automorphism which is dependent on $\hat{x}$. There is a similar automorphism

$$
h \rightarrow k_{\mathrm{S}}(\hat{x}, h)=g_{\mathrm{s}}(\hat{x})^{-1} k(\hat{x}, h) g_{\mathrm{s}}(\hat{x})
$$

appropriate for $\Sigma_{\mathrm{s}}$. In the overlap zone $\Sigma_{\mathrm{N}} \cap \Sigma_{\mathrm{s}}$ we have

$$
k_{\mathrm{N}}(\hat{x}, h)=h_{T}^{-1}(\hat{x}) k_{\mathrm{S}}(\hat{x}, h) h_{T}(\hat{x}) .
$$

These transformations $g_{\mathrm{N}}, g_{\mathrm{S}}$ are nonunique to a certain extent and this implies that $h_{T}(\hat{x})$ is also nonunique:

$$
h_{T}^{\prime}(\hat{x})=h_{\mathrm{S}}^{-1}(\hat{x})^{-1} h_{T}(\hat{x}) h_{\mathrm{N}}(\hat{x})
$$

We could make the choice that the outer automorphisms of $\mathrm{H}$ form a discrete set; then since $k_{\mathrm{N}}(\hat{x}, h)$ is to vary smoothly with $\hat{x}$ over $\Sigma_{\mathrm{N}}$ and $g_{\mathrm{N}}(N)=e$,

$$
k_{\mathrm{N}}(N, h)=h \text {. }
$$

Then by invoking the smoothness of $k_{\mathrm{N}}(\hat{x}, h)$ it follows that $h \rightarrow k_{\mathrm{N}}(\hat{x}, h)$ is an inner automorphism:

$$
k_{\mathrm{N}}(\hat{x}, h)=h_{\mathrm{N}}(\hat{x}) h h_{\mathrm{N}}^{-1}(\hat{x})
$$

We could, in turn, use this $h_{\mathrm{N}}(\hat{x})$ to change the choice of $k_{\mathrm{N}}(\hat{x}, h)$ so that

$$
k_{\mathrm{N}}(\hat{x}, h) \rightarrow k_{\mathrm{N}}^{\prime}(\hat{x}, h)=h \text {. }
$$

This is true all over $\Sigma_{\mathrm{N}}$. It remains for us to find a smooth gauge change over $\Sigma_{\mathrm{S}}$ such that

$$
k_{\mathrm{s}}^{\prime}\left(\hat{x}, h^{\prime}\right) k_{\mathrm{s}}^{\prime}(\hat{x}, h)=k_{\mathrm{S}}^{\prime}\left(\hat{x}, h^{\prime} h\right)
$$

and in the overlap zone $\Sigma_{\mathrm{N}} \cap \Sigma_{\mathrm{S}}$ it satisfies the transition relation

$$
k_{\mathrm{S}}^{\prime}(\hat{x}, h)=h_{T}^{\prime}(\hat{x}) \cdot h \cdot h_{T}^{\prime}(\hat{x})^{-1}
$$

But the transition function fails to have a unique limit as the South pole is approached. Instead, by suitable choice we hope to get 


$$
h_{T}^{\prime}(\hat{x}) \rightarrow c(\phi) \in \mathrm{H} \quad \text { as } \hat{x} \rightarrow \mathrm{S} .
$$

The transition relation becomes

$$
e(\phi) h e(\phi)^{-1}=h, \quad h \in \mathrm{H} .
$$

So unless the closed curve $c(\phi), 0 \leq \phi \leq 2 \pi$ lies in the center of $H$ the global action of $H$ cannot be defined. So if the monopole is nontrivial and $\mathrm{H}$ has a discrete center this action cannot be defined globally. It is not only that this is not a symmetry of the theory, it cannot even be defined.

\section{Color breaking: Quantum theory [9]}

Even though most of the above discussion is couched in the language of classical systems we see that this same impossibility of defining the global group $\mathrm{H}$ obtains in quantum theory. In this context we recognize that for an Abelian monopole all transformations of $\mathrm{H}$ are globally realizable.

Since the impossibility of the global application of $\mathrm{H}$ depended on the lack of commutation with the transition function $h_{T}(\hat{x})$, to this extent $h_{T}(\hat{x})$ defines the breakdown of the global group H. However it must be pointed out that two very different transition functions can describe the same monopole sector if

$$
h_{T}(\hat{x})=\mathrm{e}^{\mathrm{i} \phi T}, \quad h_{T^{\prime}}(\hat{x})=\mathrm{e}^{\mathrm{i} \phi T^{\prime}}
$$

are two transition functions such that

$$
c(\phi)=\mathrm{e}^{\mathrm{i} \phi T} \cdot \mathrm{e}^{-\mathrm{i} \phi T^{\prime}}
$$

is a closed curve which can be shrunk to zero.

As an example consider the example suggested by application to particle physics:

$$
\mathrm{H}=\left[\mathrm{SU}(3)_{\mathrm{C}} \times \mathrm{U}(1)_{\mathrm{e} . \mathrm{m} .}\right] / \mathrm{Z}_{3}=\mathrm{U}(3)
$$

For the elementary monopole we could choose

$$
h_{T}=\mathrm{e}^{\mathrm{i} T \phi} ; \quad T=\frac{1}{3}-\lambda_{8} / \sqrt{3}=\left(\begin{array}{ccc}
0 & 0 & 0 \\
0 & 0 & 0 \\
0 & 0 & 0
\end{array}\right) \text {. }
$$

The commutant of $T$ is

$$
\mathrm{K}_{T}=\mathrm{SU}(2)_{\mathrm{C}} \times \mathrm{U}(1)_{Y_{\mathrm{C}}} \times \mathrm{U}(1)_{\mathrm{e} \cdot \mathrm{m} .}
$$

where $\mathrm{SU}(2)_{\mathrm{C}}$ acts on the first two colors and $Y_{\mathrm{C}}$ is the corresponding color hypercharge. This would certainly indicate color breaking of a definite kind. However an alternative choice of the transition 
function is

$$
h_{T^{\prime}}=\mathrm{e}^{\mathrm{i} \phi T^{\prime}} ; \quad T^{\prime}=\left(\begin{array}{rrr}
-2 & 0 & 0 \\
0 & 1 & 0 \\
0 & 0 & 2
\end{array}\right) .
$$

The curve $\mathrm{e}^{\mathrm{i} T \phi} \cdot \mathrm{e}^{-\mathrm{i} T^{\prime} \phi}$ is homotopically trivial and hence $h_{T}$ and $h_{T^{\prime}}$ define the same monopole. However the globally definable transformations constitute the Abelian group

$$
\mathrm{K}_{T^{\prime}}=\mathrm{U}(1) \times \mathrm{U}(1) \times \mathrm{U}(1)
$$

So the groups $\mathrm{K}_{T}$ and $\mathrm{K}_{T^{\prime}}$ need not be the same. The homotopic classification of monopoles is too crude to tell us the full physics of symmetry breaking.

Another interesting example, again suggested by particle physics, is

$$
H=[S U(2) \times U(1)] / Z_{2}=U(2) \text {. }
$$

The transition functions $h_{T}, h_{T}$ correspond to

$$
T=\left(\begin{array}{rrr}
1 & 0 & 0 \\
0 & 0 & 0 \\
0 & 0 & -1
\end{array}\right) ; \quad T^{\prime}=\left(\begin{array}{rrr}
2 & 0 & 0 \\
0 & -1 & 0 \\
0 & 0 & -1
\end{array}\right)
$$

It is easily verified that $T$ and $T^{\prime}$ describe the same non-Abelian monopole. In this case we can construct $h(\hat{x})$ as a $3 \times 3$ matrix function of $(\theta, \phi)$ which satisfies

$$
h(0, \phi)=e ; \quad h(\pi, \phi)=h_{T}(\phi) h_{T^{\prime}}(\phi)^{-1}
$$

In this case both $\mathrm{K}_{T}$ and $\mathrm{K}_{T}$, are $\mathrm{U}(1) \times \mathrm{U}(1)$ but distinct actions.

One could ask: what if we disregarded the prohibition to extending $H$ from $\Sigma_{N}$ to all of $S^{2}$ ? What could possibly go wrong? What happens is that disallowed color transformations create configurations with infinite energy. This happens because the illegal transformations create a $\delta$-function singularity in the field strength at the South pole; and in turn, to an infinity in the total field energy. We must recognize that the old and new field strengths are not gauge transformations of each other since they differ by a $\delta$-function at the South pole.

Similar results can be demonstrated for the energy of a quantum-mechanical particle in a color monopole field.

\section{Gauge automorphisms and canonical implementability}

Having recognized the startling result that color symmetry could be broken in the presence of non-Abelian monopoles we ought to be concerned with other symmetries including Lorentz invariance. 
We should also carefully distinguish between automorphisms that leave the relations unaltered in form and genuine symmetries realized by canonical transformations. This we proceed to do.

Let us consider a theory involving gauge fields whose potentials are denoted by $W_{\mu}$ and matter fields which we take to be spinor fields $\psi$. The Lagrangian of the system in interaction and the equations of motion are invariant under a large group $\mathrm{G}$ of automorphisms:

$$
W_{\mu} \rightarrow h W_{\mu} h^{-1}+h \partial_{\mu} h^{-1} ; \quad \psi \rightarrow D(h) \psi
$$

where $D(h)$ is the matrix realization of the gauge group $\mathrm{G}$ on $\psi$. The transformation $h(x)$ is required to be smooth but otherwise unrestricted; it need not go to any specific limit as $x$ goes to infinity.

Even in usual field theories not all automorphisms are canonically implementable: in quantum field theory this means the impossibility of defining unitary operators generating such transformations while in classical field theories this implies the impossibility of canonical transformations realizing the automorphism. The canonically implementable transformations form a subgroup $\mathrm{G}^{(\mathrm{U})}$ of $\mathrm{G}$. We may consider this as the spontaneous breakdown of $G$ to $G^{(U)}$. Among $G^{(U)}$ there is a subgroup $G_{0}$ which is generated by the Gauss law operator

$$
D \cdot E+J_{0}
$$

where $E$ is the color electric field and $J_{0}$ is the color charge density. Since the Gauss law operator vanishes on any physical state, on physical states the group $G_{0}$ is trivial. We may therefore identify the nontrivial group

$$
\mathrm{G}_{1}=\mathrm{G}^{(\mathrm{U})} / \mathrm{G}_{0}
$$

as the global symmetry of the theory. In $\mathrm{QCD}$, for example, $\mathrm{G}_{0}$ goes to unity at infinity while the connected component $\mathrm{G}_{0}^{(\mathrm{U})}$ of $\mathrm{G}^{(\mathrm{U})}$ include transformations which go to any constant at infinity independent of the angle along which we go to infinity. Then the physical group $G_{0}^{(U)} / G_{0}$ is isomorphic to the global color group which was the starting point of the gauge theory.

Following Hanson, Regge and Teitelboim [10] we recognize the need to smear the Gauss law operator and consider objects like

$$
g(\Lambda)=\int \mathrm{d}^{3} x \operatorname{Tr}\left(\Lambda(x)\left(D \cdot E+J_{0}\right)(x)\right)
$$

to vanish on physical states. The test functions $A(x)$ are arbitrary for finite $x$, but the asymptotic properties of $\Lambda(x)$ should be properly defined. We shall require that $\Lambda(x)$ is to be so chosen that $g(\Lambda)$ is a differentiable functional of $\Lambda(x)$ in the sense that

$$
\delta g(\Lambda)=-\int \mathrm{d}^{3} x \operatorname{Tr}(\boldsymbol{E} \cdot \boldsymbol{D} \Lambda)
$$

This requires, in turn, 


$$
\lim _{r \rightarrow \infty} \int \mathrm{d} \Omega r^{2} \cdot \operatorname{Tr}(\Lambda \hat{x} \cdot \delta \boldsymbol{E})=0
$$

Such a requirement of differentiable functionals is necessary for the Poisson brackets to be properly defined and to obtain the correct field equations from the action principle.

Along with $g(\Lambda)$ we define also the closely related charge operator

$$
Q(M)=\int \mathrm{d}^{3} x \operatorname{Tr}\left(M J_{0}-\boldsymbol{E} \cdot \boldsymbol{D} M\right)
$$

which differs from $g(M)$ only by a surface term provided a partial integration can be done; otherwise they are distinct. The allowed phase space functionals $Q(M)$ generate the connected component $\mathrm{G}_{0}^{(\mathrm{U})}$ of the implementable group $\mathrm{G}^{(\mathrm{U})}$.

\section{Gauge theories: Standard sector}

For orientation let us first consider an Abelian gauge theory. If the charge and current distributions are of compact support the $r^{-2}$ piece of both electric and magnetic fields are odd functions of $\hat{x}$ so that

$$
\begin{aligned}
& \boldsymbol{E}=\boldsymbol{e}^{(-)}(\hat{x}) / r^{2}+\mathrm{O}\left(1 / r^{3}\right) \\
& \boldsymbol{B}=\boldsymbol{b}^{(-)}(\hat{x}) / r^{2}+\mathrm{O}\left(1 / r^{3}\right) \\
& \boldsymbol{A}=\boldsymbol{a}^{(+)}(\hat{x}) / r+\partial_{i} \chi(\boldsymbol{x})+\mathrm{O}\left(1 / r^{2}\right)
\end{aligned}
$$

where $e^{(-)}, b^{(-)}$are odd and $\boldsymbol{a}^{(+)}$even in $\hat{x}$. Then the allowed sets of $\Lambda(x)$ are of the form

$$
\Lambda=\Lambda^{(-)}(\hat{x})+\mathrm{O}(1 / r)
$$

while for $M(x)$ it is

$$
M=\beta+\Lambda^{(-)}(\hat{x})+\mathrm{O}(1 / r), \quad \beta=\text { constant } .
$$

The quotient group $\mathrm{G}_{0}^{(\mathrm{U})} / \mathrm{G}_{\mathrm{U}}^{(\infty)}=\mathrm{U}(1)$ with generators

$$
Q(\beta)=\beta \int \mathrm{d}^{3} x J_{0}
$$

Among the gauge transformations only the odd part of $\chi(x)$ can be changed by the acceptable gauge transformations. The gauge transformations including $\chi^{(+)}(\hat{x})$ are spontaneously broken.

Similar considerations apply for the nonmonopole sectors of non-Abelian gauge theories. We need 


$$
\begin{aligned}
& \boldsymbol{E}=\frac{1}{r^{2}} h(x) \boldsymbol{e}^{(-)}(\hat{x}) h(x)^{-1}+\mathrm{O}\left(\frac{1}{r^{3}}\right) \\
& \boldsymbol{B}=\frac{1}{r^{2}} h(x) \boldsymbol{b}^{(-)}(\hat{x}) h(x)^{-1}+\mathrm{O}\left(\frac{1}{r^{3}}\right) \\
& \boldsymbol{A}=\frac{1}{r} h(x) \boldsymbol{a}^{(+)}(\hat{x}) h(x)^{-1}+h(x) \partial h(x)^{-1}+\mathrm{O}\left(\frac{1}{r^{3}}\right) \\
& {\left[a_{j}^{(+)}(\hat{x}), a_{h}^{(+)}(\hat{x})\right]=0,}
\end{aligned}
$$

with $h(x)$ unrestricted at infinity. Differentiability of the Gauss law functional demands

$$
\Lambda(x)=h(x) \Lambda^{(-)}(\hat{x}) h(x)^{-1}+\mathrm{O}(1 / r)
$$

and

$$
\left[\Lambda^{(-)}(\hat{x}), \Lambda^{(-)}\left(\hat{x}^{\prime}\right)\right]=\lambda^{(-)}(\hat{x})
$$

with any odd $\lambda^{(-)}$. On $M(x)$ the requirement is

$$
M(x)=h(x) N h(x)^{-1}+\mathrm{O}(1 / r)
$$

with $N$ any constant Lie algebra element.

\section{Monopole sector of gauge theory}

When monopole sectors are considered we have to reanalyze the situation in further detail: so we restrict considerations to the Georgi-Glashow model. The spherically symmetric connection furnishes the vector potential

$$
W=\frac{1}{2} \tau \cdot \hat{x} \partial \tau \cdot \hat{x}+\mathrm{O}\left(1 / r^{2}\right) \text {. }
$$

In this case the $r^{-2}$ terms in $\boldsymbol{E}$ and $\boldsymbol{B}$ are even and the leading term in $\boldsymbol{W}$ odd in contrast to the nonmonopole sector,

$$
\begin{aligned}
& \boldsymbol{E}=\frac{1}{r^{2}} h(x) e^{(+)}(\hat{x}) h(x)^{-1}+\mathrm{O}\left(\frac{1}{r^{2}}\right) \\
& \boldsymbol{B}=\frac{1}{r^{2}} h(x) \boldsymbol{b}^{(+)}(\hat{x}) h(x)^{-1}+\mathrm{O}\left(\frac{1}{r^{2}}\right) \\
& \boldsymbol{A}=\frac{1}{r} h(x) \boldsymbol{a}^{(-)}(\hat{x}) h(x)^{-1}+h(x) \partial h(x)^{-1} .
\end{aligned}
$$


The test functions $\Lambda(x)$ and $M(x)$ are required to be

$$
\begin{aligned}
& \Lambda(x)=h(x) \Lambda^{(+)}(\hat{x}) h(x)^{-1}+\mathrm{O}(1 / r) \\
& M(x)=h(x)\left(\alpha^{(\infty)}+\alpha^{(-)}(\hat{x})\right) h(x)^{-1}+\mathrm{O}(1 / r) .
\end{aligned}
$$

\section{Question of Lorentz invariance}

Let us now examine the question of Lorentz invariance. Due to the "mixing of spin and isospin" the rotation generators contain color operators. The rotation and boost generators are respectively the integral of the Poynting vector and the moment of the energy density. The Poisson bracket of these generators do not close amongst themselves but contain supplementary terms:

$$
\left\{J_{k}, K_{j}\right\}=\varepsilon_{k j l} K_{l}+g\left(\chi_{k j}\right)
$$

where $\chi_{k j}$ depends on the fields. Such terms arise in multiple Poisson brackets also. If such additional term had $\chi$ which were among the allowed test functions for the Gauss law $\Lambda$ then these $g(\chi)$ would vanish on physical configurations and consequently we would have implemented Lorentz invariance. We can calculate

$$
\left\{K_{j},\left\{K_{k}, J_{l}\right\}\right\}=\varepsilon_{k i n} \varepsilon_{j n m} J_{m}+g\left(\chi_{j k l}\right)
$$

In the monopole sector $\chi_{j k l}$ do not vanish at infinity. So if we restrict our attention to only a Gauss law form in which $g(\Lambda)$ reduce to unity at infinity, we would be forced to conclude that Lorentz invariance breaks in the presence of monopoles. So it is essential that we consider a more stringent Gauss law which permits $\Lambda$ which do not vanish at infinity.

There is much work needed to clarify our notion of invariances and transformations within gauge field theories and more generally with dynamical systems with infinite number of degrees of freedom. Physics has moved into a phase where topologies and geometrical considerations are playing a crucial role. The distinction of canonically implementable transformations and automorphisms is an essential physical consideration.

The research reported in this paper is based on collaboration with A.P. Balachandran, G. Marmo, N. Mukunda and J.S. Nilsson. Some of the results have not been reported elsewhere yet. I thank Giuseppe Marmo for his interest and assistance in the preparation of this paper. This work was supported by the Department of Energy under Grant No. DE-FG05-85ER40200 at The University of Texas at Austin.

\section{References}

[1] E.C.G. Sudarshan and N. Mukunda, Classical Dynamics: A Modern Perspective (John Wiley, New York, 1974).

[2] F. Zaccaria, E.C.G. Sudarshan, J.S. Nilsson, N. Mukunda, G. Marmo and A.P. Balachandran, Phys. Rev. D27 (1983) 2327.

[3] P.A.M. Dirac, Proc. Roy. Soc. London A133 (1931) 60; Phys. Rev. 74 (1948) 817; M.N. Saha, Phys. Rev. 75 (1949) 1968. 
[4] T.T. Wu and C.N. Yang, Phys. Rev. D12 (1975) 3843; D12 (1975) 3845; D14 (1976) 437.

[5] A.P. Balachandran, G. Marmo, B.S. Skagerstam and A. Stern, Nucl. Phys. B162 (1980) 385; B164 (1980) 427;

A.P. Balachandran, G. Marmo, N. Mukunda, J.S. Nilsson, E.C.G. Sudarshan and F. Zaccaria, Phys. Rev. D29 (1984) 2919;

J.L. Friedman and R.D. Sorkin, Phys. Rev. D20 (1979) 2511;

A.P. Balachandran, G. Marmo, B.S. Skagerstam and A. Stern, Lecture Notes in Physics 188 (Springer, Berlin, 1983).

[6] S.K. Wong, Nuovo Cimento 65A (1970) 689

[7] S. Coleman, The Magnetic Monopole Fifty Years Later, in: Proc. E. Majorana Summer School 1981, ed. A. Zichichi (Plenum, N.Y., 1983).

[8] For an introduction to forms and fibre bundles, A. Trautman, Differential Geometry for Physicists (Bibliopolis, Naples, 1984).

[9] A.P. Balachandran, G. Marmo, N. Mukunda, J.S. Nilsson, E.C.G. Sudarshan and F. Zaccaria, Phys. Rev. Lett. 50 (1983) 1553; Phys. Rev. D29 (1984) 2919; 2936;

A. Abouelnood, Nucl. Phys. B226 (1984) 309; Phys. Lett. 125B (1983) 467;

P. Nelson and A. Monohor, Phys. Rev. Lett. 50 (1983) 943;

P. Nelson and S. Coleman, Nucl. Phys. B237 (1984) 1;

P.A. Horvathy and H.H. Rownsley, Comm. Math. Phys. 96 (1984) 497;

C.N. Yang, Phys. Rev. D1 (1970) 2360;

P.A. Horvathy and J.H. Rownsley, Internal symmetries of non-Abelian gauge field configurations (Preprint CPT-C.N.R.S., Luminy, 1984); Fibre bundles, Monopoles and Internal Symmetries (preprint CPT-C.N.R.S., Luminy, 1985).

[10] Hanson, T. Regge and C. Teitelboim, Constrained Hamiltonian Systems, Acc. Naz. dei Lincei (Rome, 1976). 